Muro de la investigación, 2018: 3(2), agosto-diciembre,

ISSN: 2523-2886

DOI: https://doi.org/10.17162/rmi.v3i2.1162

\title{
Relación entre dependencia a videojuegos y habilidades sociales en estudiantes de una institución educativa estatal de Lima Este
}

\author{
Sislay Marcia Alave Mamani, ${ }^{1}$ Sara Nelbith Pampa Yupanqui ${ }^{2}$ \\ Universidad Peruana Unión, Naña Perú ${ }^{12}$
}

Recibido: 13 de febrero de 2018

Aceptado: 16 de junio de 2018

\begin{abstract}
Resumen
El presente estudio describe la relación entre la dependencia a los videojuegos y las habilidades sociales en los estudiantes de una Institución Educativa estatal de Lima-este. La muestra se compuso de 375 adolescentes de ambos sexos en dicho centro educativo. Como instrumentos se utilizó el Test de dependencia a videojuegos de Marcos y Cholíz (2012) y la Escala de Habilidades Sociales (EHS) de Goldstein (1978), adaptada en el Perú por Ambrosio Tomás en 1995. Los resultados de la investigación revelan una correlación débil, negativa y significativa entre la dependencia a videojuegos y las habilidades sociales en general ( $r h o=-.132 \mathrm{p}<0.05)$; por otro lado, se encontró una relación significativa en las dimensiones de "habilidades alternativas a la agresión" (rho= -.209; $\mathrm{p}<0.05)$ y "habilidades relacionadas con el estrés" (rho= $-.108 ; \mathrm{p}<0.05)$. En suma, mientras se presenten niveles bajos de dependencia a videojuegos existirán más recursos para relacionarse adecuadamente o viceversa.
\end{abstract}

Palabras clave: Dependencia a videojuegos, habilidades sociales, adicción, adolescentes.

\section{Relationship between dependence on video games and social skills in students of a State School in East Lima}

\begin{abstract}
This research work describes the relationship between videogame dependence and social skills in students of a state school in Lima-east. The sample consisted of 375 adolescents of both sexes in said educational center. As instruments are the Choliz y Marco' Video Game Dependence Test (2012) and Goldstein's Social Skills Scale (EHS) (1978), adapted in Peru by Ambrosio Tomás in 1995. The results of the research reveal a correlation weak, negative and significant between video game dependence and social skills in general (rho $=-.132 \mathrm{p}<0.05$ ); on the other hand, a significant relationship was found in the dimensions of "alternative skills to aggression" (rho $=-.209 ; \mathrm{p}<0.05)$ and "stress-related skills" (rho $=-.108 ; \mathrm{p}<0.05)$. In sum, while there are low levels of dependence, there are video games plus resources to relate to limitations or vice versa.
\end{abstract}

Keywords: Dependence on videogames, social skills, addiction, adolescents.

\footnotetext{
${ }^{1}$ E-mail: sislayalave@upeu.edu.pe

${ }^{2}$ E-mail: sara.nelbith@upeu.edu.com
} 


\section{Introducción}

Una característica común en los hábitos de consumo de los adolescentes a nivel mundial es el excesivo empleo del tiempo en los videojuegos, lo cual es un problema cada vez más frecuente y creciente cada año. En Norteamérica, alrededor de 200 millones de usuarios consume videojuegos casi 13 horas a la semana (Newzoo, 2015). En España, los videojuegos han alcanzado el segundo contenido digital más destacado (Fundación Orange, 2014), lo cual representa un negocio rentable para los programadores y distribuidores, cuyo objetivo es obtener ganancias y exorbitantes ventas de las consolas y accesorios de juego que fabrican (Asociación Española de Videojuegos [AEVI], 2015).

Por su parte, Chóliz y Marco (2011) encontraron que el 5.7\% de 562 adolescentes evaluados en Valencia (España) presentaron síntomas de dependencia a videojuegos; el $10 \%$ presentó abuso de ellos, y el $84.2 \%$ presento uso no patológico. Igualmente, en Madrid, el 29.7\% de 1710 escolares evaluados presentaron un cuadro de adicción a videojuegos (Labrador \& Villadangos, 2010).

En el Perú, 2 de cada 10 adolescentes entre 11 y 18 años presentan casos de dependencia a los videojuegos; y 7 de cada 10 abusa de ellos (Vallejos \& Capa, 2010). Por cierto, un informe (2014) del Instituto Nacional de Salud Mental Honorio Delgado Hideyo Noguchi, reveló que-entre los años 2012 y 2013 - acudieron a consulta 297 pacientes nuevos con problemas de adicción a internet y ludopatía. Asimismo, el mayor porcentaje que padece de adicción a los videojuegos lo constituyen los adolescentes y adultos jóvenes.

Por otro lado, el Ministerio de Salud (MINSA) demostró durante el año 2013 el incremento de las atenciones de menores adictos a los videojuegos al 25\% en hospitales públicos en relación al año 2012 (citado por La República, 2014). Por su parte, Arnau, Falla, y Jiménez (2011) agregan que casi la mitad (41\%) de 164 adolescentes pertenecientes a las regiones costa y selva del Perú presentaban problemas de adicción a los videojuegos, en una cantidad de 4 u 8 horas al día.

Con respecto a este fenómeno, Echeburua y De Corral (2010) afirman que el videojuego constituye una afición patológica que origina dependencia en el individuo, afectando sus actividades e intereses. Por su parte, Chóliz y Marco (2011) definen a la dependencia del videojuego como el uso excesivo o compulsivo de este, al grado de interferir negativamente con la vida personal y las actividades diarias del sujeto. Por consiguiente, la Clínica de la Universidad de Navarra (2015) señaló la manera eventual e "inocente" que ocurre al inicio del empleo de videojuegos, cuya incidencia aumenta hasta 
alcanzar una práctica diaria. Además, este uso incontrolable desarrolla una desorganización grave en la vida del adolescente.

Tejeiro, Pelegrina, y Gómez, (2009) señalan el peligro emocional y social que posee la excesiva dependencia a los videojuegos, en el caso de convertir al usuario en un ser aislado, debido a que la actividad de juego suele ser solitaria. Esto induce al individuo a reducir sus interacciones amicales, y poniendo en riesgo sus habilidades sociales. Asimismo, Caballo (2007) afirma que el efecto de la exposición continua a los videojuegos se manifiesta en sentimientos, actitudes, deseos, opiniones y derechos de un modo inadecuado a la situación, sin respetar la de los demás. De igual modo Anderson (2008) realizó un estudio en el que se obtuvo como resultado que un grupo de personas expuestas a videojuegos de contenido violento presentaron indicadores de agresividad, lo cual es una manifestación inadecuada de la interacción social.

Asimismo, Griffiths y Meredith (2009) consideran que jugar compulsivamente produce serios efectos negativos, como dejar de realizar actividades de la vida cotidiana, no atender a las relaciones sociales prioritarias, prefiriendo realizar sus actividades de manera solitaria y perdiendo la atención a su entorno social (Betancourt, González, \& Martínez, 2013).

Elaborar este estudio sobre el tema se considera relevante, debido a que en la adolescencia ocurren cambios emocionales y físicos, surge la búsqueda de identidad y la construcción de la propia personalidad (Weissmann, 2011); en la gran mayoría de casos, existe una dinámica de configurar los jóvenes su autodeterminación, autonomía e individualización, por lo que recurren a medios en los que supuestamente encuentran refugio (Sierra, Reyes, \& Córdoba, 2010). Por su parte Parolari (2005) afirma que en la etapa de la adolescencia es común la "imitación" de personajes, lenguajes, formas de comportamiento, y demás, con el fin de lograr pertenencia a un determinado grupo social, lo que cumple la necesidad de pertenecer a algo y ser reconocido por sus grupos pares.

Por todo lo expuesto en líneas anteriores, la presente investigación intenta determinar la relación entre dependencia a videojuegos y habilidades sociales de estudiantes de una institución educativa estatal de Lima Este.

\section{Método y materiales}

\section{Diseño}

Este trabajo presenta un diseño no-experimental, pues no existió manipulación de las variables. Es de corte transversal, porque los datos fueron tomados en un solo 
momento en la historia. Asimismo, el alcance de la investigación es de tipo correlacional, porque cuantifica la relación entre variables y su meta es responder a las causas de los eventos físicos o sociales (Hernández, Fernández, \& Baptista, 2010).

\section{Participantes}

El muestreo fue de tipo no probabilístico intencional. La investigación fue desarrollada en una institución educativa ubicada en Lima Este. La muestra estuvo conformada por 375 adolescentes, de ambos géneros. Sus edades oscilaron entre 12 y 18 años.

\section{Instrumentos}

Para este trabajo se utilizó el Test de Dependencia a Videojuegos (TDV), elaborado por Marcos y Cholíz (2011). Este modelo fue adaptado al Perú sin modificaciones por Edwin Sala y Merino César, en el año 2017. La función de este instrumento es diagnosticar el grado de dependencia de los videojuegos en individuos; consta de 25 ítems y presenta una consistencia interna elevada. Recibió una validez de constructo mediante el análisis factorial exploratorio y el análisis factorial confirmatorio donde se corroboró la presencia de cuatro dimensiones.

Los primeros 14 ítems se responden mediante una escala tipo Likert que oscila entre 0 y 4, referida a la frecuencia ( 0 , nunca; 1, rara vez: 2 a veces; 3 con frecuencia y 4, casi siempre), y en los 11 ítems restantes se pregunta por el grado "de acuerdo" o "desacuerdo," a través de una escala tipo Likert, que oscila entre 0 y 4 ( 0 , totalmente de acuerdo; 1 , un poco en desacuerdo; 2 , neutral; 3 , un poco de acuerdo; 4 , totalmente desacuerdo), sobre un conjunto de afirmaciones relacionadas con los videojuegos. El cuestionario está compuesto por cuatro dimensiones: abstinencia (malestar que se presenta cuando el sujeto no puede jugar con videojuegos y uso de los mismos para aliviar sus problemas psicológicos), abuso y tolerancia (jugar progresivamente más que al principio y de forma excesiva), problemas asociados por los videojuegos (consecuencias negativas del uso excesivo de los videojuegos) y dificultad de control (dificultades para dejar de jugar, a pesar de que no sea funcional ni adecuado hacerlo en ese momento o situación). Muestra aceptables propiedades, el índice de alfa de Cronbach del conjunto de ítems es de 0.94 por lo que se le considera un instrumento con características psicométricas satisfactorias. 
La Lista de Chequeo de Habilidades Sociales de 1978 fue adaptada en el Perú por Ambrosio Tomás en 1995. Su objetivo principal es comprobar las deficiencias y competencias que tiene una persona en sus habilidades sociales y cómo las usa. Se compone de 50 ítems, agrupados en seis áreas: habilidades sociales primarias, habilidades sociales avanzadas, habilidades relacionadas con los sentimientos, habilidades alternativas a la agresión, habilidades para hacer frente al estrés y habilidades de planificación; los cuales se presentan en una escala reconocida del 1 al 5.

En cuanto a validez y confiabilidad, Tomás. (1995) halló correlaciones significativas ( $\mathrm{p}<.05, .01 \mathrm{y} .001)$, por lo que el instrumento quedó intacto, con todos sus ítems completos, sin eliminar alguno. La prueba test-retest obtuvo una " $r$ " $=0.6137$ y una " $\mathrm{t} "=3.011$, la cual es muy significativa al $\mathrm{p}<.01$. Finalmente, el coeficiente Alpha de Cronbach obtuvo un Alpha Total "rtt" $=0.9244$. Todos estos valores demostraron la precisión y estabilidad de la Lista de Chequeo de Habilidades Sociales.

\section{Resultados}

Niveles de dependencia a videojuegos

En la tabla 1, se observa que el $40.5 \%$ de escolares encuestados presenta un nivel moderado de habilidades sociales, lo que indica su proceso de formar capacidades y destrezas para el contacto interpersonal y para la solución de problemas de índole socioemocional. E1 39.7\% evidencia niveles altos en esta variable, pues poseen adecuadas habilidades sociales. En cambio, el 19.8\%, presenta niveles bajos en el desarrollo de este tipo de habilidades, lo que significa su dificultad para establecer y mantener relaciones interpersonales.

En cuanto a las dimensiones se observa que el $45.5 \%$ de la población de estudio presenta niveles altos en las "habilidades sociales primarias": saben cómo iniciar una conversación y mantener sus relaciones interpersonales. Por otro lado, el 55\% posee niveles moderados en las "habilidades sociales avanzadas," su proceso de relacionarse satisfactoriamente en entornos sociales está en aumento. En cuanto a la dimensión "habilidades sociales relacionadas con los sentimientos," el $45.0 \%$ posee niveles moderados, lo que equivale a afirmar que casi la mitad de la población evaluada se encuentra en desarrollo aún de reflejar, expresar y reconocer sentimientos. En la dimensión "habilidades sociales alternativas a la agresión," el 46.1\% presenta niveles altos, lo que significa que son capaces de manejar sus emociones evitando conflictos y 
mostrándose asertivos; mientras que el $15.9 \%$ presenta un nivel bajo en esta dimensión, es decir, no tiene autocontrol ni empatía al momento de enfrentarse a situaciones de enfado. Respecto de "habilidades sociales para afrontar el estrés", el 42.7\% presenta niveles moderados, lo que significa que están en proceso de ser tolerantes a la frustración. Finalmente, el $41.0 \%$ posee niveles moderados en las "habilidades sociales de planificación", es decir, planifican objetivos organizados, tratando de culminar sus trabajos.

Tabla 1

Nivel de Dependencia a videojuegos y sus dimensiones

\begin{tabular}{lcccccc}
\hline & \multicolumn{2}{c}{ Bajo } & \multicolumn{2}{c}{ Moderado } & \multicolumn{2}{c}{ Alto } \\
\cline { 2 - 7 } & $\mathrm{n}$ & $\%$ & $\mathrm{n}$ & $\%$ & $\mathrm{n}$ & $\%$ \\
\hline Dependencia a videojuegos & 163 & 45.5 & 107 & 29.9 & 88 & 24.6 \\
Abstinencia & 170 & 47.5 & 108 & 30.2 & 80 & 22.3 \\
Abuso y tolerancia & 189 & 52.8 & 80 & 22.3 & 89 & 24.9 \\
Problemas asociados a los & 170 & 47.5 & 102 & 28.5 & 89 & 24.9 \\
videojuegos & 167 & 46.6 & 107 & 29.9 & 84 & 23.5 \\
Dificultad en el control & & & & &
\end{tabular}

\section{Niveles de habilidades sociales}

De igual forma, en la tabla 2 se observa que el $40.5 \%$ de escolares presenta un nivel moderado de habilidades sociales, situación que indica su desarrollo en formar capacidades y destrezas para el contacto interpersonal y para la solución de problemas de índole socioemocional. El 39.7\% evidencia niveles altos en esta variable, es decir, posee adecuadas habilidades sociales. En cambio, el 19.8\% presenta niveles bajos en el desarrollo de estas habilidades, indicando dificultad para establecer y mantener relaciones interpersonales y poseen complicaciones o para afrontar el estrés o la planificación de actividades.

Por otro lado, el $45.5 \%$ de la población de estudio presenta niveles altos en las "habilidades sociales primarias," es decir, saben cómo iniciar una conversación y mantener sus relaciones interpersonales. Asimismo, el 55\% posee niveles moderados en las "habilidades sociales avanzadas" lo que indica que están en proceso de relacionarse satisfactoriamente en entornos sociales. La dimensión "habilidades sociales relacionadas con los sentimientos" posee niveles moderados, pues casi la mitad de la población estudiada (el 45.0\%) está en proceso de reflejar, expresar y reconocer sentimientos. El $46.1 \%$ de los encuestados presenta niveles altos en la dimensión "habilidades sociales alternativas a la agresión," lo que indica su capacidad en manejar sus emociones evitando 
conflictos y mostrándose asertivos; mientras que el $15.9 \%$ presenta un nivel bajo en esta dimensión, no posee autocontrol ni empatía al momento de enfrentarse a situaciones de enfado. Respecto a las "habilidades sociales para afrontar el estrés", el 42.7\% presenta niveles moderados, lo que significa que están en proceso de ser tolerantes a la frustración. Finalmente, el $41.0 \%$ posee niveles moderados en las "habilidades sociales de planificación", es decir, planifican objetivos organizados, tratando de culminar sus trabajos.

Tabla 2

Nivel de habilidades sociales en escolares

\begin{tabular}{lcccccc}
\hline & \multicolumn{2}{c}{ Bajo } & \multicolumn{2}{c}{ Moderado } & \multicolumn{2}{c}{ Alto } \\
\cline { 2 - 7 } & $\mathrm{N}$ & $\%$ & $\mathrm{n}$ & $\%$ & $\mathrm{n}$ & $\%$ \\
\hline $\begin{array}{l}\text { Habilidades sociales } \\
\begin{array}{l}\text { Habilidades sociales } \\
\text { primarias }\end{array}\end{array}$ & 71 & 19.8 & 145 & 40.5 & 142 & 39.7 \\
$\begin{array}{l}\text { Habilidades sociales } \\
\text { avanzadas }\end{array}$ & 34 & 13.1 & 148 & 41.3 & 163 & 45.5 \\
$\begin{array}{l}\text { Habilidades sociales } \\
\text { relacionadas con los }\end{array}$ & 72 & 20.1 & 161 & 45.0 & 125 & 34.9 \\
$\begin{array}{l}\text { sentimientos } \\
\text { Habilidades sociales } \\
\text { alternativas a la agresión }\end{array}$ & 57 & 15.9 & 136 & 38.0 & 165 & 46.1 \\
$\begin{array}{l}\text { Habilidades sociales para } \\
\text { afrontar el estrés }\end{array}$ & 75 & 20.9 & 153 & 42.7 & 130 & 36.3 \\
$\begin{array}{l}\text { Habilidades sociales de } \\
\text { planificación }\end{array}$ & 116 & 32.4 & 150 & 41.9 & 92 & 25.7 \\
\hline
\end{tabular}

\section{Prueba de normalidad}

En la tabla 3 se puede apreciar la posible distribución normal de las variables. Con el objetivo de realizar los análisis de comparación y contrastación que se han planteado, se procedió ante todo a realizar la prueba de bondad de ajuste para determinar si las variables presentan distribución normal. Esta tabla expone los resultados obtenidos a través de la prueba de bondad de ajuste de Kolmogorov- Smirnov (K-S). Se observa que en la variable "habilidades sociales" dos dimensiones no presentan distribución normal debido a que la significancia asintótica es menor a 0.05 ; lo mismo ocurre con la variable “dependencia a videojuegos.” Para esto, se utilizó una estadística no paramétrica para el análisis estadístico. 
Tabla 3

Prueba de bondad de ajuste a la curva normal para las variables de estudio

\begin{tabular}{llcrcc}
\hline Instrumento & Variable & Media & D.E. & K-S & P \\
\hline \multirow{5}{*}{$\begin{array}{l}\text { Habilidades } \\
\text { sociales }\end{array}$} & Habilidades sociales global & 172.05 & 28.52 & .033 & .200 \\
& Habilidades sociales primarias & 27,03 & 5.45 & .055 & .011 \\
& Habilidades sociales avanzadas & 20.73 & 3.94 & 0.76 & .000 \\
& Habilidades sociales alternativas a la & 32.47 & 6.49 & .065 & .001 \\
& agresión & & & & \\
& $\begin{array}{l}\text { Habilidades sociales para afrontar el } \\
\text { estrés }\end{array}$ & 39.77 & 8.15 & 0.42 & .200 \\
Dependencia de videojuego & 26.30 & 23.31 & .130 & .000 \\
\hline
\end{tabular}

\section{Correlación de variables}

Correlación de dependencia a videojuegos y habilidades sociales

En la tabla 4, el coeficiente de correlación Spearman indica una relación negativa y significativa entre dependencia a videojuegos y habilidades sociales global $(r=-, 132$, $\mathrm{p}<0.01$ ); es decir, a mayor nivel de dependencia a videojuegos menor nivel de habilidades sociales se produce. En cuanto a la correlación entre las dimensiones de habilidades sociales y la dependencia a videojuegos, se evidenció que existe una correlación débil, inversa y significativa con la dimensión habilidades sociales alternativas a la agresión $(r=-, 209, p<0.05)$, es decir, cuando los niveles de dependencia a videojuegos son bajos, existe más recursos para solucionar o afrontar situaciones estresantes o conflictos interpersonales y viceversa. La misma correlación existe entre la dimensión "habilidades sociales para afrontar el estrés" $(\mathrm{r}=-, 108, \mathrm{p}<0.05)$ y "dependencia a videojuegos;" lo que muestra que, mientras más altos son los niveles de desarrollo de estas habilidades (posesión de mecanismos de afrontamiento apropiados ante situaciones de crisis, entre otras) los niveles de dependencia a videojuegos son menores y viceversa. Por otro lado, no se encontró relación significativa entre dependencia de videojuegos y las dimensiones primeras habilidades sociales $(r=-, 052, \mathrm{p}>0.05)$ y habilidades sociales avanzadas $(\mathrm{r}=-$ ,069, p>0.05), es decir, estas variables se desarrollan de manera desvinculada en la población de estudio.

Tabla 4

Coeficiente de correlación entre dependencia a videojuegos y habilidades sociales

\begin{tabular}{lcc}
\hline Habilidades sociales & \multicolumn{2}{c}{ Dependencia a videojuegos } \\
\cline { 2 - 3 } & Rho & $\mathrm{p}$ \\
\hline Habilidades sociales global &,- 132 &, 013
\end{tabular}


Habilidades sociales primarias

Habilidades sociales avanzadas

Habilidades sociales alternativas a la agresión

**la correlación es significativa al nivel 0.01

\section{Discusión}

La presente investigación busca determinar la relación entre dependencia a videojuegos y habilidades sociales en estudiantes de una institución educativa estatal de Lima Este. En primer lugar, se resalta la diferencia en el patrón de uso entre varones y mujeres: los de género masculino son la población que más incidencia presenta en el consumo de videojuegos (Alonqueo \& Rehbein, 2008). Asimismo, estos medios presentan contenidos muy violentos, ocasionando en los adolescentes la exhibición de conductas agresivas como respuesta o solución ante los problemas (Etxeberría, 2011). Por su parte, Chóliz y Marco (2011) confirman la relación patológica entre la conducta y la actividad de los juegos en red, actividad que se convierte en una práctica desmedida y excesiva, con efectos negativos en el adolescente. Asimismo, Tejeiro, Pelegrina y Gómez (2009) describen la conducta solitaria en los consumidores recurrentes de videojuegos, lo que ocasiona el deterioro o estancamiento de las habilidades sociales de los adolescentes.

Respecto a la hipótesis general, los resultados muestran una correlación débil, negativa y significativa entre dependencia a videojuegos y habilidades sociales en general $(\mathrm{rho}=-.132 \mathrm{p}<0.05)$. Esto afirma que, al presentarse niveles bajos de dependencia de videojuegos existen más recursos para relacionarse apropiadamente, dando solución adecuada a situaciones estresantes o conflictos interpersonales y viceversa. Por otro lado, mientras existan niveles altos de dependencia a videojuegos, se presentarán niveles bajos o inadecuados de habilidades sociales.

En este sentido, Ramos (2014) observa que el tiempo desmedido al uso de videojuegos, perjudica y altera el proceso de las dinámicas de socialización, pues la exposición continua y exagerada de videojuegos influye en el desarrollo y la práctica de habilidades sociales (Lillo, Morales \& Romero, 2013). La actividad de juego es imperiosa e insociable, el individuo se aleja de sus contactos interpersonales debido a que se reducen sus interacciones, lo que produce que se haga menos social y pone en riesgo sus habilidades de relación (Tejeiro, Pelegrina, \& Gómez, 2009).

En otro orden de cosas, se evidenció que existe una relación negativa y significativa entre dependencia a videojuegos y las dimensiones "habilidades sociales alternativas a la agresión" ( $(\mathrm{hho}=-.209 ; \mathrm{p}<0.05)$ y las "habilidades sociales para afrontar 
el estrés" (rho= -.108; p<0.05). La primera dimensión corresponde al manejo de las emociones y la asertividad. Su relación con la dependencia a videojuegos explica que, al existir niveles elevados de dependencia de estos juegos, los adolescentes presentan niveles bajos o inadecuados de empatía, manejos emocionales y autocontrol. Esto puede desencadenar comportamientos agresivos y prepotentes al momento de solucionar los conflictos interpersonales (Goldstein et al., 1989). Al respecto, Etxeberría (2011) encontró que la exposición a videojuegos violentos guarda relación directa con las conductas agresivas $\mathrm{y}$, al mismo tiempo, disminuye los comportamientos prosociales y de empatía.

Respecto a la segunda dimensión, "tolerancia ante la frustración," los resultados evidenciaron que los niveles altos de dependencia de videojuegos producen niveles bajos o inadecuados de afrontamiento al estrés; no obstante, los adolescentes que dedican horas prolongadas a estos juegos, poseen dificultades para manejar situaciones embarazosas, y situaciones de riesgo (Goldstein et al., 1989). Al respecto, Anderson, Gentile y Buckley (2007) afirmaron que el uso de videojuegos se constituye un poderoso inductor de conductas violentas y agresivas. Asimismo, Pérez y Prado (2014) afirman que los videojuegos de contenido violento conducen a reacciones violentas como gritar, golpear, utilizar un lenguaje vulgar y también hostilidad (cf. Puma \& Vilca, 2014).

En cambio, no se halló relación significativa entre dependencia de videojuegos y la dimensión "habilidades sociales primarias" ( $\mathrm{rho}=-.0 .52 ; \mathrm{p}>0.05)$; esta dimensión no está influenciada por la dependencia de videojuegos. Por otra parte, la dimensión "habilidades sociales avanzadas" ( $r h o=-.0 .69 ; \mathrm{p}>0.05)$ tampoco muestra relación directa con la dependencia a videojuegos.

Finalmente, se explica la realidad que el ser humano necesita reconocimiento en lo colectivo, respeto ante los demás y ocupar un puesto en la sociedad; en consecuencia, llevar a cabo relaciones interpersonales satisfactorias. Todo ello afirma que el aspecto social es una competencia básica y avanzada en la vida del ser humano (Armida \& Candoval, 2012). En conclusión, el hecho que los encuestados dependan o no de los videojuegos no guarda ningún vínculo o relación con el desarrollo que estos presenten de sus primeras habilidades sociales y habilidades sociales avanzadas.

\section{Referencias}

Alonqueo, P., \& Rehbein, L. (2008). Usuarios habituales De videojuegos: una aproximación inicial. Revista Última Década, 29, 11-27. 
https://doi.org/10.4067/S0718-22362008000200002

Anderson, C., Gentile, D., \& Buckley, K. (2007). Violent Video Game Effects on Children and Adolescents: Theory, Research, and Public Policy. New York: Oxford University.

Anderson, C., Sakamoto, A., Gentile, D., Ihori, N., Shibuya, A., Yukawa, y Kobayashi, K. (2008). Longitudinal Effects of Violent Video Games on Aggression in Japan and the United States. Pediatrics, 122(5), 1067-1072.

Apaza, J., \& Bedregal, J. (2014). Uso de videojuegos relacionado a las habilidades sociales en adolescentes de la I.E. Peruano del Milenio Almirante Miguel Grau Cayma. Universidad Nacional de San Agustín.

Armida, S., \& Candoval, M. (2012). Psicología del Desarrollo Humano I. Dgep (4th ed.). México.

Arnau, J., Falla, G., \& Jiménez, A. (2011). Los juegos en linea en adolescentes y jóvenes un estudio cuanti-cualitativo descriptivo y análitico. CEDRO, 2-35.

Asociación Española de Videojuegos - AEVI. (2015). Estudio de Hábitos y Usos de los Videojuegos. Recuperado de: http://www.aevi.org.es/adese-presenta-el-estudio-dehabitos-y-usos-de-los-videojuegos/

Caballo, V. (2007). Manual de evaluacion y entrenamiento de habilidades sociales. (S. X. de E. Editores, Ed.) (7ma ed.). España.

Chóliz, M., \& Marco, C. (2011). Patrón de uso y dependencia de videojuegos en infancia y adolscencia. TT - Pattern of use and dependence on video games in infancy and adolescence. Anales de Psicología, 27(2), 418-426.

Echeburua, E., \& De Corral, P. (2010). Adicción a las nuevas tecnologías y a las redes sociales en jóvenes: un nuevo reto. Adicciones, 22(2), 91-96. Recuperado de: http://www.adicciones.es/files/91-96 editorial echeburua.pdf

Etxeberría, F. (2011). Videojuegos violentos y agresividad. Pedagogia Social Revista Interuniversitaria, (18), 31. https://doi.org/10.7179/PSRI_2011.18.03

Fundacion Orange. (2014). Informe anual sobre el desarrollo de la sociedad de la información en España. Fundación Orange. España. Retrieved from https://www.proyectosfundacionorange.es/docs/eE2014/Informe_eE2014.pdf

Goldstein, A., Sprafkin, R., Gershaw, J., \& Klein, P. (1989). Habilidades sociales y autocontrol en la adolescencia. (M. Roca, Ed.) (2da ed.). Barcelona.

Griffiths, M., \& Meredith, A. (2009). Videogame addiction and its treatment. Journal of Contemporary Psychotherapy, 39(4), 247-253. Hernandez, R., Fernandez, C., \& 
Baptista, M. (2010). Metodología de la investigación. Metodología de la investigación (5th ed.). Mexico.

Instituto Nacional de Salud Mental Honorio Delgado - Hideyo Noguchi. (2014). Expertos en salud mental exhortan a los padres mayor vigilancia a sus hijos en el acceso a los videojuegos porque pueden generar adicción.

Labrador, F., \& Villadangos, S. (2010). Menores y nuevas tecnologías: Conductas indicadoras de posible problema de adicción. Psicothema, 22(2), 180-188.

Llavero, I. (2015). Videojuegos y los niños. Universidad Politecnica de Valencia. Retrieved from https://www.cun.es/chequeos-salud/infancia/videojuegos-ninos

Martínez, M. (2009). Dimensiones Básicas de un Desarrollo Humano Integral. Revista de La Universidad Bolivariana, 8(23), 119-138.

Martínez, P., Betancourt, D., \& González, A. (2013). Uso de videojuegos, agresión, sintomatología depresiva y violencia intrafamiliar en adolescentes y adultos jóvenes. Revista Colombiana de Ciencias Sociales, 4(2), 167-180.

Monjas, M. (2000). La timidez en la infancia y en la adolescencia. Madrid: Pirámide. Recuperado de: https://www.casadellibro.com/libro-la-timidez-en-la-infancia-y-enla-adolescencia/9788436814392/694566

Papalia, D., Wendkos, S., \& Duskin, R. (2009). Psicologia del Desarrollo, 697.

Perez, K., \& Prado, A. (2014). Uso de videojuegos y conductas agresivas en escolares del Colegio Santa Marìa, Sector Jerusalèn, distrito La Esperanza, Trujillo, 2014. Universidad Privada Antenor Orrego.

Puma, I., \& Vilca, T. (2014). Uso de videojuegos violentos relacionado al nivel de agresividad en adolescentes de la I. E. Gran Unidad Escolar Mariano Melgar. Universidad Nacional de San Agustin.

Ramos, F. (2014). Influencia de los videojuegos on-line en la sociabilidad del adolescente. Universidad de la República Uruguay.

República, L. (2014). Adicción a los videojuegos es una enfermedad que la familia debe ayudar a superar. Recuperado de: http://larepublica.pe/sociedad/764262-adicciona-los-videojuegos-es-una-enfermedad-que-la-familia-debe-ayudar-a-superar

Tejeiro, R., Pelegrina, M., \& Gómez, J. (2009a). Efectos psicosociales de los videojuegos. Comunicación, 1(7), 235-250.

Tejeiro, R., Pelegrina, M., \& Gómez, J. L. (2009b). Efectos psicosociales de los videojuegos. Comunicación, 7, 235-250.

Vallejos, M., \& Capa, W. (2010). Video juegos: adicciones y factores predictores. 
Avances Psicologicos, 18(1), 103-110.

Weissmann, P. (2011). Adolescencia. Revista Iberoamericana de Educación, (16815653), 1-8. 